\title{
On Analytical Methods in Neuroblastoma Detection
}

\author{
R. Martínez-Díaz, ${ }^{1}$ J. Balaguer, ${ }^{2}$ L. M. Sánchez Ruiz, ${ }^{1}$ P. Bello, ${ }^{2}$ \\ V. Castel, ${ }^{2}$ and G. Perís-Fajarnés ${ }^{1}$ \\ ${ }^{1}$ Universitat Politècnica de València, Camino de Vera s/n, 46022 Valencia, Spain \\ ${ }^{2}$ Hospital La Fe, Bulevar Sur s/n, 46026 Valencia, Spain \\ Correspondence should be addressed to L. M. Sánchez Ruiz; lmsr@mat.upv.es
}

Received 11 September 2013; Accepted 7 October 2013

Academic Editor: Constantin Udriste

Copyright (C) 2013 R. Martínez-Díaz et al. This is an open access article distributed under the Creative Commons Attribution License, which permits unrestricted use, distribution, and reproduction in any medium, provided the original work is properly cited.

\begin{abstract}
Nonlinear dynamics of cancer recurrence are known to be governed by several factors as initial tumour size, number of metastatic sites, or quantity of drug resistant cells. The precise extent and location of tumours are very important factors so quantitative and consistent methods of evaluation are needed to assess reponse to patient therapy. Whole-body ${ }^{123} I$-metaiodobenzylguanidine (mIBG) scintigraphy is used as primary medical image modality to detect neuroblastoma tumours due to its high specificity and sensitivity. However, current oncological guidelines are based on qualitative observer-dependent analysis. This fact makes it difficult to compare results of scintigraphies taken at different moments during therapy or at different institutions. In this paper, we review analytical methods used in neuroblastoma detection and propose an observer-independent method to quantitatively analyse a ${ }^{123}$ I-mIBG scintigraphy.
\end{abstract}

\section{Introduction}

Nonlinear dynamics of cancer recurrence are governed by a balance between several factors such as initial tumor size, mutation rates, and growth kinetics of drug-sensitive and resistance cells [1]. In recent years, there has been an increasing interest for mathematical models in tumour growth, by taking into account the temporal evolution which can be controlled by slight changes in the sequence of tumour images. Based on these images, many theoretical models have been proposed [2-6].

Neuroblastoma is the third most common malignant solid tumor of childhood. It is metastatic or with high risk for recurrence in nearly 50\% of cases [7]; therefore, successful planning of individual patient therapy requires precise delineation of the extent of the neuroblastoma.

Anatomic imaging methods, such as Computerized Tomography (CT) and Magnetic Resonance Imaging (MRI), are most useful for the evaluation of the primary tumor mass and nearby involved lymph nodes. Functional imaging radioactive tracers, such as ${ }^{123} \mathrm{I}$-mIBG, ${ }^{18} \mathrm{~F}$-EDG, and ${ }^{99} \mathrm{~m} T \mathrm{c}$-MDP, are used to assess the extent of disease and to search for distant metastases [8].
In particular, neuroblastoma's cells take up and metabolize norepinephrine, so its analogue metaiodobenzylguanidine (mIBG) is an ideal specific agent for imaging. ${ }^{123} \mathrm{I}^{-}$ mIBG is a gamma emitting radionuclide developed in the early 1980 s to visualise tumours [9]. ${ }^{123}$ I-mIBG scintigraphy has been in clinical use for more than 20 years for diagnostic assessment. Current radiological guidelines for image acquisition with ${ }^{123} I$-mIBG in [10] define that scanning is to be performed 24 hours after its injection. A single or multiple head gamma camera with a large field of view is necessary to acquire planar images. The patient should be placed in the supine position to perform a whole-body imaging. Gamma camera scans the whole patient body counting gamma photons detected at each position. The scan produces a planar image where each pixel's intensity represents gamma photons detected at this position in patient's body so that white peaks could be observed at patient's mIBG avid tissues (e.g., tumour, metastases, etc.). Each whole-body scan includes two images, the front and the rear scan of the patient's body.

To date, radiological guidelines in [10] define a qualitative interpretation criterion of image which has been defined in terms of visual differences between pathological uptake 
pattern against physiological (normal) pattern. Following these qualitative guidelines, physicians can perform a good evaluation of a given image in terms of clear positive findings or clear negative results. But they are unable to compare results of a given scintigraphy with other previous ones to evaluate the stage of disease when visual differences between scans are not so evident.

A major drawback to the diagnosis of successive scans is that they display variations in intensity, due to the difficulty of maintaining the same exact conditions of the patient and the gamma camera in every scanning session. This variation in intensity is caused by changes in the accumulation of radioisotopes during each examination, the image quality of the gamma camera, the volume of water intake, the amount of urine excreted, or the response to drug treatment of each patient.

Current oncological studies [11] are focused on finding some observer-independent method allowing of comparison of the results between scintigraphies that may help to stratify patient response to therapy. In this paper, we introduce a simple procedure for quantitative observer-independent measurement of uptake level in a single whole-body ${ }^{123} \mathrm{I}$ mIBG scintigraphy.

This paper is structured as follows. In Section 2, we detail the proposed observer-independent measurement method. Experimental results are provided in Section 3, and conclusions are drawn in Section 4.

\section{Materials and Methods}

${ }^{123}$ I-mIBG scintigraphies were acquired in human studies by Nuclear Medicine Unit of Hospital La Fe following guidelines described in [10] after a slow intravenous injection of ${ }^{123} \mathrm{I}^{-}$ mIBG (dose: $100 \mu \mathrm{Ci} / \mathrm{Kg}$ ) and using a scintillation camera set to a photopeak equal to the principal gamma photon emitted, that is, of $159 \mathrm{KeV}$. A minimum of 250,000 and a maximum of 500,000 photon counts were obtained for each front and rear view.

The group of mIBG scans from each patient were read in a blinded fashion by two trained clinicians without knowledge of the interpretation of the original report made by the nuclear medicine physicians with good correlation.

Each scintigraphic image is a representation of the radioactive distribution inside the body. Each pixel contains a discrete value, which is related to the number of gamma photons detected within the period of acquisition. These discrete values follow a statistical Poisson distribution due to the random nature of radioactive disintegration.

In other words, the number of discrete arrivals over a period of time is not constant but is modelled by Poisson's equation as follows:

$$
P(X=k)=\frac{\mu^{k} e^{-\mu}}{k !},
$$

where $P(X=k)$ is the probability that the number $X$ of detected photons is equal to $k$ and $\mu$ is the mean value (which is unknown) of the distribution.
This formula implies in daily nuclear medicine practice that the number of photon counts has to be increased in order to reduce the effect of Poisson noise. This can be achieved by increasing the acquisition time, which increases the patient motion and decreases camera throughput, or increasing the amount of administered radioactive material, which will lead to serious patient's health problems. Given these limitations, image processing techniques are needed to work without increasing photon counts.

First attempts to quantify uptake in regions of interest (ROI) in scintigraphies were made in 1973. In [12], an uptake measurement of ${ }^{99} \mathrm{mTc}$ in thyroid studies based on the number of gamma photons detected (counts) was proposed. The main idea was to calculate the ratio between counts in ROI divided by the total body counts.

Heart-to-mediastinal (H/M) uptake ratio derived from mIBG scintigraphy has been used as a quantitative measurement of uptake with success to monitor response to medical treatment in areas such as cardiac sympathetic imaging [13]. This measure of specific to nonspecific uptake is intrinsically very simple: the ratio of the counts per pixel in two regions of interest (ROIs), one drawn around the heart and the other drawn in the midline upper thorax. This simplicity has contributed to the widespread use of the $\mathrm{H} / \mathrm{M}$ ratio for comparing different populations. However, there is great variation of $\mathrm{H} / \mathrm{M}$ ratio among publications from various institutions.

We propose also a very simple approach based on the uptake ratio between neuroblastoma tumours and the rest of tissues to standarize the measurement procedure and to minimize variation in measurements between institutions.

Let us denote by $I$ the front (or rear) view of a whole-body ${ }^{123} I$-mIBG scintigraphy of size $m \times n$ pixels. Let $x_{i j} \in[0,1]$ be the intensity of pixel at coordinates $(i, j)$ in image $I$ that represents radionuclide uptake by tissue (photon counts) at these coordinates. Since we are interested in measuring the uptake inside the patient's body, we will only take into account the set $I^{+}=\left\{(i, j) \in I: x_{i j}>0\right\}$.

And let $\bar{I}$ and $\sigma_{I}$ be the mean and the standard deviation of intensity in $I$, given, respectively, by (2) and (3) as

$$
\begin{gathered}
\bar{I}=\frac{\sum_{(i, j) \in I^{+}} x_{i j}}{\#\left\{I^{+}\right\}}, \\
\sigma_{I}=\sqrt{\frac{1}{\#\left\{I^{+}\right\}} \sum_{(i, j) \in I^{+}}\left(x_{i j}-\bar{I}\right)^{2}} .
\end{gathered}
$$

In order to be able to compare the uptake value $x_{i j}$ at pixel $(i, j)$ with uptake value $x_{i j}^{\prime}$ at the same patient's body coordinates in another scintigraphy, we need both values to be transformed into the same normalized scale.

We will transform the intensity level at each pixel $x_{i j}$ into its normalized uptake value $U_{I}(i, j)$ with $(4)$ as

$$
U_{I}(i, j)=\max \left(\frac{x_{i j}-\bar{I}}{\sigma_{I}}, 0\right),
$$


TABLE 1: $\bar{U}_{I}$ (background) and measurements of regions of interest at a series of five scintigraphies from $T_{1}$ to $T_{5}$ of patient P4 (Figure 1).

\begin{tabular}{lcccrr}
\hline Regions & $T_{1}$ & $T_{2}$ & $T_{3}$ & $T_{4}$ & 0,3 \\
\hline $\bar{U}_{I}$ (background) & 0,25 & 0,22 & 0,26 & 1,01 & 1,6 \\
$\bar{U}_{I}$ (Right Femur) & 0,3 & 0,27 & 1,3 & 1,39 & 0,29 \\
$\bar{U}_{I}$ (Liver) & 1,43 & 1,35 & 0,26 & 0,3 & 0,94 \\
$\bar{U}_{I}$ (Left Tumor) & 1,3 & 0,22 & 2,4 & 1,68 & 0,29 \\
$\bar{U}_{I}$ (Right Tumor) & 1,35 & 0,22 & 2,35 \\
\hline
\end{tabular}

and we call it the normalized uptake value at coordinates $(i, j)$ referenced to $\bar{I}$. Notice that we are only interested in measuring those $x_{i j}$ values that are greater than $\bar{I}$ because they are the relevant uptake points. Uptake at $x_{i j}$ values lower than $\bar{I}$ has a value of $U_{I}(i, j)=0$.

Once every pixel in scintigraphy is transformed into its normalized uptake value based on its standard deviation from mean $\bar{I}$, we are able to take measurements that are comparable with the next scintigraphies of the same patient.

When measuring uptake at any body point, image is quantified by areas, but not by pixels. A single pixel may have a high scintigraphy value, but it is not taken into account because noise exists in the original image.

For noise reduction, we need to replace raw counts at region with processed counts having reduced noise. This can be achieved by smoothing counts over neighboring pixels. The smoothed area is a weighted average, in which raw counts at point $R_{j}$ are replaced by processed counts $Q_{i}$ given by (5) where the weights $w_{i j}$ are chosen to provide a smoothing scheme and are normalized by the sum rule in (6) as

$$
\begin{aligned}
& Q_{i}=\sum_{j} w_{i j} R_{j}, \\
& \sum_{j} w_{i} \equiv 1 \quad \forall i .
\end{aligned}
$$

Therefore, when measuring any image region of interest $\mathrm{ROI}_{k}$ (e.g., liver, tumour, etc.), we will use weights equal to $1 / k$ where $k$ is the number of pixels in the region. Then the uptake measurement, $\bar{U}_{I}\left(\mathrm{ROI}_{k}\right)$, of any region of interest is defined as the average of the positive normalized uptakes at $(i, j) \in \mathrm{ROI}_{k}$; that is,

$$
\bar{U}_{I}\left(\mathrm{ROI}_{k}\right)=\frac{\sum\left\{U_{I}(i, j):(i, j) \in \mathrm{ROI}\right\}}{\#\{\mathrm{ROI}\}} .
$$

\section{Results and Discussion}

We took measurements in a test set of whole-body ${ }^{123} I^{-}$ mIBG scintigraphies of annoymous patients. This experimental set contains 23 whole-body ${ }^{123} I$-mIBG scintigraphies belonging to 19 children under 14 labeled patients from P1 to P19 suffering from neuroblastoma taken with different gamma cameras.

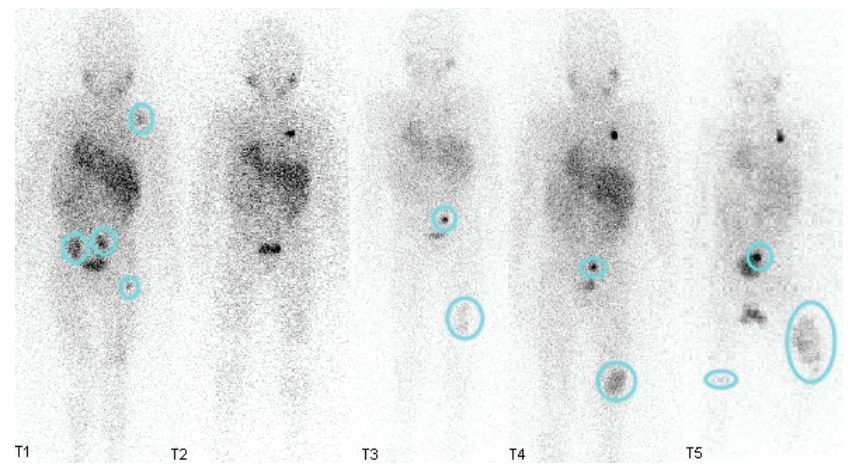

FIGURE 1: A series of five original rear view ${ }^{123} I$-mIBG test samples of patient P4 from $I_{\mathrm{P} 4 T_{1}}$ to $I_{\mathrm{P} 4 T_{5}}$ with diagnosed tumour areas selected inside circles.

We were interested in calculating the uptake present at the whole patient's body frame which we call $\bar{U}_{I}$ (background) and is defined as follows:

$$
\bar{U}_{I}(\text { background })=\frac{\sum\left\{U_{I}(i, j):(i, j) \in I^{+}\right\}}{\#\left\{I^{+}\right\}} .
$$

At every whole-body scintigraphy in test set, physicians labeled each tumour region $R_{k}$ in each view (rear and front) and measured $\bar{U}_{I}\left(R_{k}\right)$ with (7) applied to tumour pixels of each $R_{k}$. In case a tumour was visible in both views, it had two measurements, the $\bar{U}_{I}\left(R_{k}\right)$ referenced to $\bar{I}$ of the front view and another measurement in the rear view.

We also calculated the whole-body $I$ background uptake mean $\bar{U}_{I}$ (background) in both views (rear and front) with (8) and $\bar{U}_{I}$ (liver).

A comparison of the measurement results of $\mathrm{P} 4$ patient's ${ }^{123}$ I-mIBGs (only the rear view for simplicity) is shown in Figure 2 and Table 1 where we may appreciate that there exists a threshold of reference at $\bar{U}_{I}$ (background) that remains close to 0.3 in all five P4 scintigraphies, mainly in $T_{4}$ and $T_{5}$. Relation between $\bar{U}_{I}$ (background) and each $\bar{U}_{I}$ (tumour) may be used as an evidence of tumour uptake evolution. Furthermore, $\bar{U}_{I}$ (background) values at both views are similar in all the $\mathrm{P} 4$ patient's mIBGs.

In Figure 3 and Table 2, evolution of $\bar{U}_{I}$ (background) values in scintigraphies $T_{1}$ and $T_{2}$ is consecutive in time of patients $\mathrm{P} 4, \mathrm{P} 10, \mathrm{P} 11$, and P16. In all four patients, 


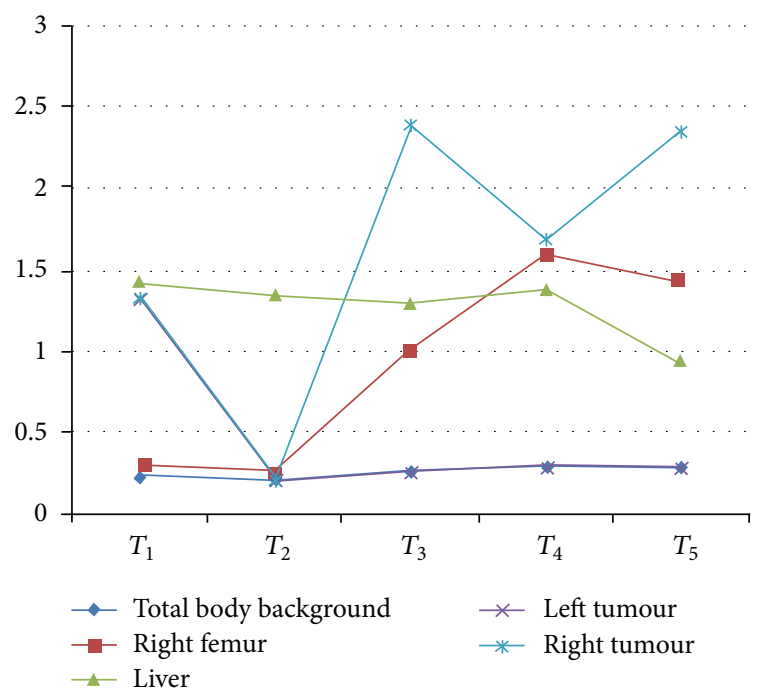

FIgURE 2: Evolution of $\bar{U}_{I}$ (regions) at tumour regions and $\bar{U}_{I}$ (background).

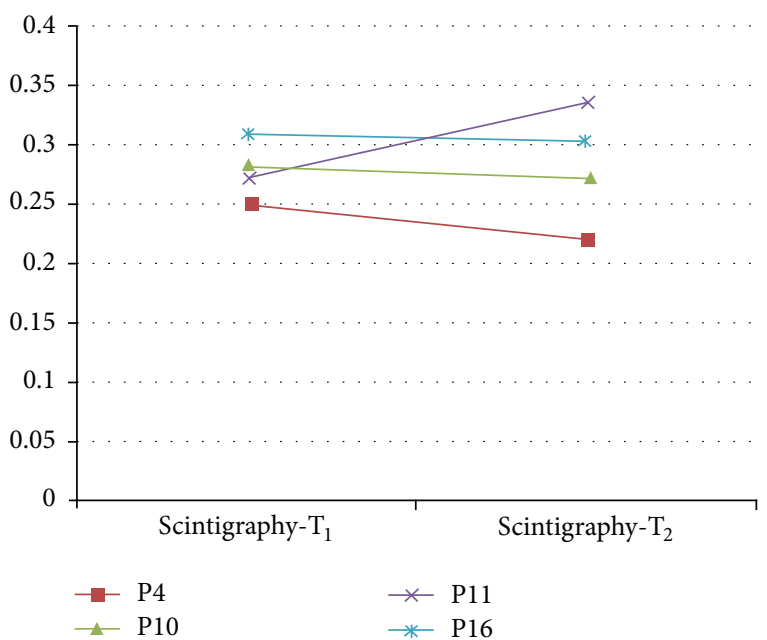

FIGURE 3: Evolution of $\bar{U}_{I}$ (background) values in front views of scintigraphies $T_{1}$ and $T_{2}$ consecutive in time of patients P4, P10, P11, and P16.

TABLE 2: $\bar{U}_{I}$ (background) at scintigraphies $T_{1}$ and $T_{2}$ of patients $\mathrm{P} 4$, $\mathrm{P} 10, \mathrm{P} 11$, and P16.

\begin{tabular}{lcc}
\hline $\bar{U}_{I}$ (background) & $T_{1}$ & $T_{2}$ \\
\hline P4 & 0,25 & 0,22 \\
P10 & 0,2816 & 0,271 \\
P11 & 0,2727 & 0,337 \\
P16 & 0,3072 & 0,3034 \\
\hline
\end{tabular}

$\bar{U}_{I T_{2}}$ (background) of $T_{2}$ scintigraphy is close to $\bar{U}_{I T_{1}}$ (background) of previous scintigraphy $T_{1}$, specially in patients P10 and P16. $\bar{U}_{I}$ (background) may be used as a threshold of reference to compare results.

Comparison between all patients' results is shown in Figure 4 . We show in red squares $\bar{U}_{I}$ (background) of rear

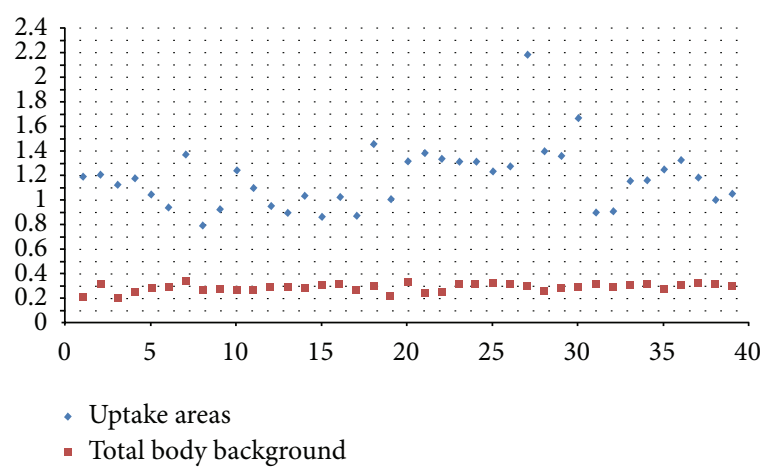

FIgURE 4: Comparison between all patients' results. In red squares, $\bar{U}_{I}$ (background) of front and rear views of each scintigraphy of each patient and in blue diamonds $\bar{U}_{I}$ (informed tumours) and $\bar{U}_{I}$ (livers).

and front views of the scintigraphies of each patient and in blue diamonds $\bar{U}_{I}$ (informed tumours) and $\bar{U}_{I}$ (livers). In the whole test set, $\bar{U}_{I}$ (background) remains close to 0.3 , in both rear and front views, that will allow us to compare results between different patients.

\section{Conclusions}

A simple observer-independent method for measuring the uptake level of ${ }^{123} I$-mIBG in any body region of children has been presented. This technique enables us to measure the relative amount of uptake with a quantitative and objective unit based on standard deviation of the uptake area. The lesion grey level is used as an uptake measurement evidence. Any region of interest could be measured in any other patient and compared with the estimated normal grey level. The larger the standard deviation value of grey level is at a given region, the greater the possibility of tumour existence is.

Preliminary results in test set show that the relationship between the background uptake mean and the tumours uptake mean may be used as evidence of a patient's tumours uptake evolution due to the fact that background uptake mean tends to be constant in every analysed ${ }^{123} I-\mathrm{mIBG}$ scintigraphy of the same patient at both rear and front views.

Moreover, the results of the whole-body background uptake mean obtained at rear and front views are similar in the whole test set, even though different gamma cameras were used on different patients and years. This indicates that the method may be useful to compare results between different patients and even between different medical institutions.

These quantitative measurements add more descriptive information of tumours that may be included in models of nonlinear dynamics of cancer recurrence to study correlation between uptake and tumour relapse.

\section{Conflict of Interests}

The authors declare that there is no conflict of interests regarding the publication of this paper. 


\section{Acknowledgments}

The authors would like to acknowledge the important contribution of doctors A. Rivas and A. Cañete of the Hospital La Fe in Valencia (Spain) for the development of this work.

\section{References}

[1] J. Foo and K. Leder, "Dynamics of cancer recurrence," Annals of Applied Probability, vol. 23, no. 4, pp. 1437-1468, 2013.

[2] N. Bellomo and M. Delitala, "From the mathematical kinetic, and stochastic game theory to modelling mutations, onset, progression and immune competition of cancer cells," Physics of Life Reviews, vol. 5, no. 4, pp. 183-206, 2008.

[3] C. Bianca and M. Delitala, "On the modelling of genetic mutations and immune system competition," Computers \& Mathematics with Applications, vol. 61, no. 9, pp. 2362-2375, 2011.

[4] C. Bianca and M. Pennisi, "Immune systems modelling by topdown and bottom-up approaches," International Mathematical Forum, vol. 7, no. 3, pp. 109-128, 2012.

[5] C. Cattani and A. Ciancio, "Separable transition density in the hybrid model for tumor-immune system competition," Computational and Mathematical Methods in Medicine, vol. 2012, Article ID 610124, 6 pages, 2012.

[6] C. Cattani, A. Ciancio, and A. d'Onofrio, "Metamodeling the learning-hiding competition between tumours and the immune system: a kinematic approach," Mathematical and Computer Modelling, vol. 52, no. 1-2, pp. 62-69, 2010.

[7] W. P. Mueller, E. Coppenrath, and T. P. Uger, "Nuclear medicine and multimodality imaging of pediatric neuroblastoma," Pediatric Radiology, vol. 43, no. 4, pp. 418-427, 2013.

[8] S. E. Sharp, M. T. Parisi, M. J. Gelfand, G. A. Yanik, and B. L. Shulkin, "Functionalmetabolic imaging of neuroblastoma," Quarterly Journal of Nuclear Medicine and Molecular Imaging, vol. 57, pp. 6-20, 2013.

[9] M. Nakajo, B. Shapiro, and J. Copp, "The normal and abnormal distribution of the adrenomedullary imaging agent $\mathrm{m}$-[i131]iodobenzylguanidine (i-131 mibg) in man: evaluation by scintigraphy," Journal of Nuclear Medicine, vol. 24, no. 8, pp. 672-682, 1983.

[10] E. Bombardieri, F. Giammarile, C. Aktolun et al., "131i/123iMetaiodobenzylguanidine (mibg) scintigraphy: procedure guidelines for tumour imaging," European Journal of Nuclear Medicine and Molecular Imaging, vol. 37, no. 12, pp. 2436-2446, 2010.

[11] K. K. Matthay, B. Shulkin, R. Ladenstein et al., "Criteria for evaluation of disease extent by 123i-metaiodobenzylguanidine scans in neuroblastoma: a report for the International neuroblastoma risk group (inrg) task force," British Journal of Cancer, vol. 102, no. 9, pp. 1319-1326, 2010.

[12] M. N. Maisey, T. K. Natarajan, P. J. Hurley, and H. N. Wagner Jr., "Validation of a rapid computerized method of measuring ${ }^{99} \mathrm{mTc}$ pertechnetate uptake for routine assessment of thyroid structure and function," Journal of Clinical Endocrinology and Metabolism, vol. 36, no. 2, pp. 317-322, 1973.

[13] W. Chen, Q. Cao, and V. Dilsizian, "Variation of heart-tomediastinal ratio in ${ }^{123} \mathrm{I}-\mathrm{miBG}$ cardiac sympathetic imaging: its affecting factors and potential corrections," Current Cardiology Reports, vol. 13, no. 2, pp. 132-137, 2011. 


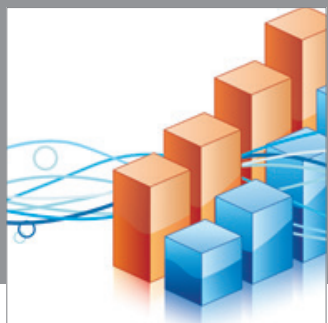

Advances in

Operations Research

mansans

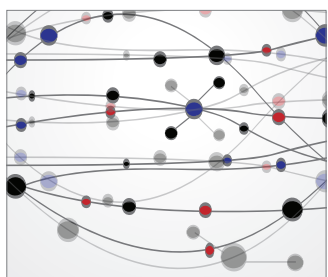

The Scientific World Journal
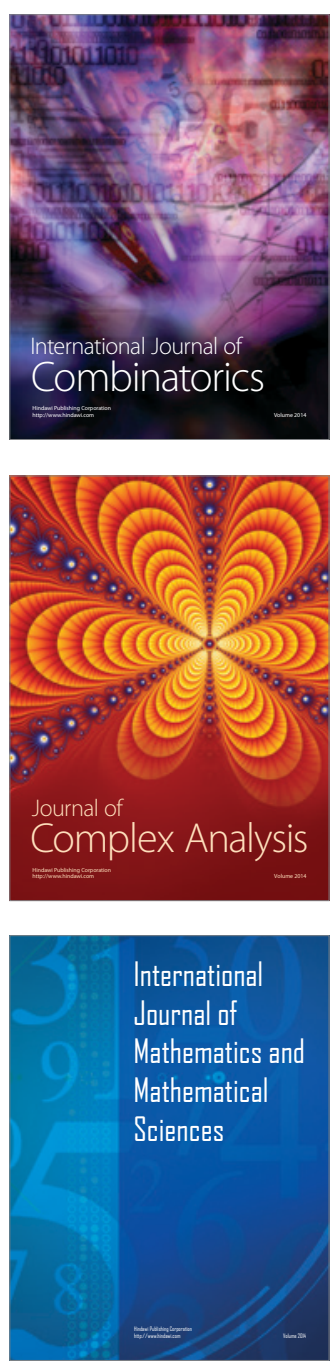
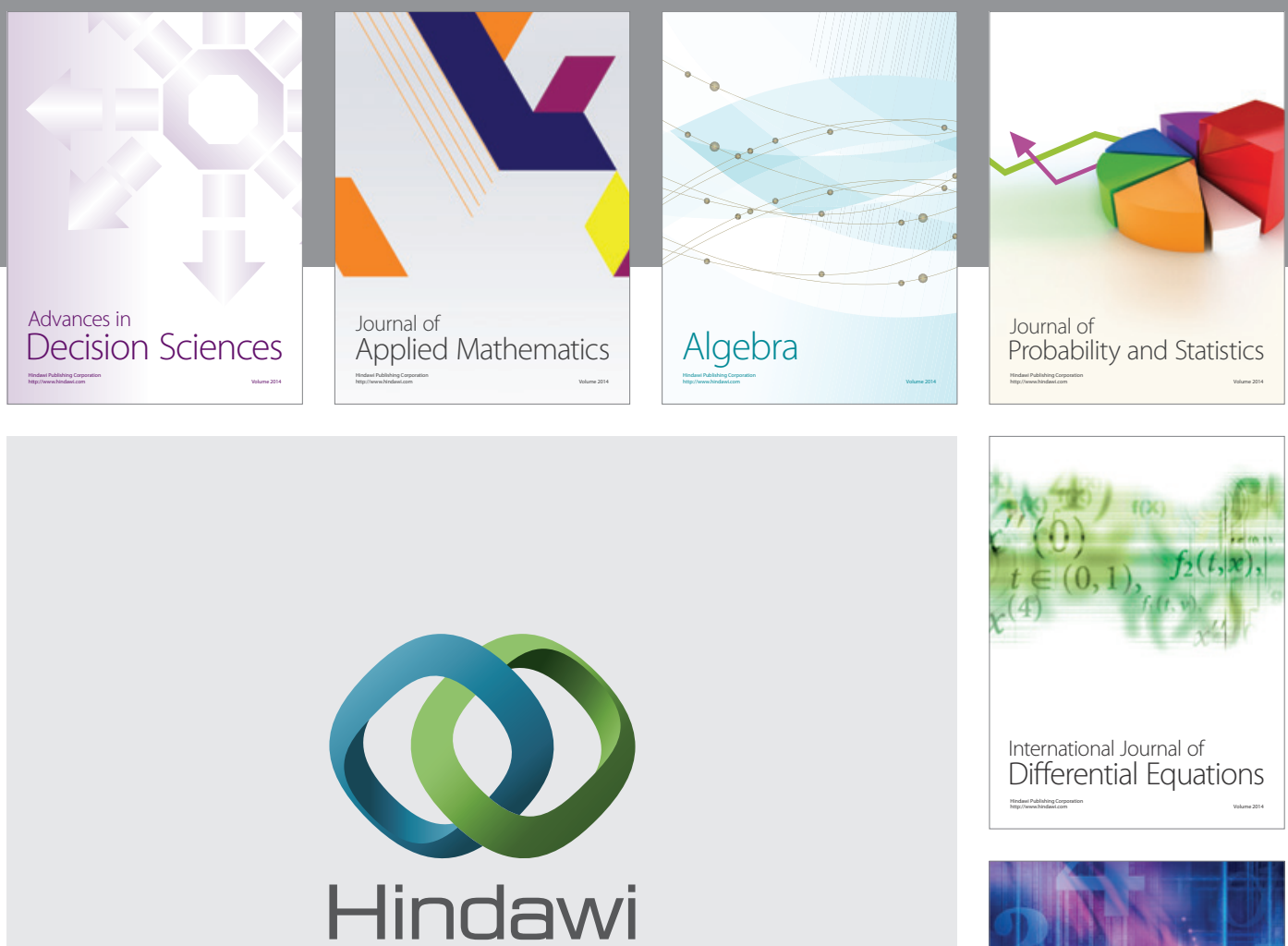

Submit your manuscripts at http://www.hindawi.com
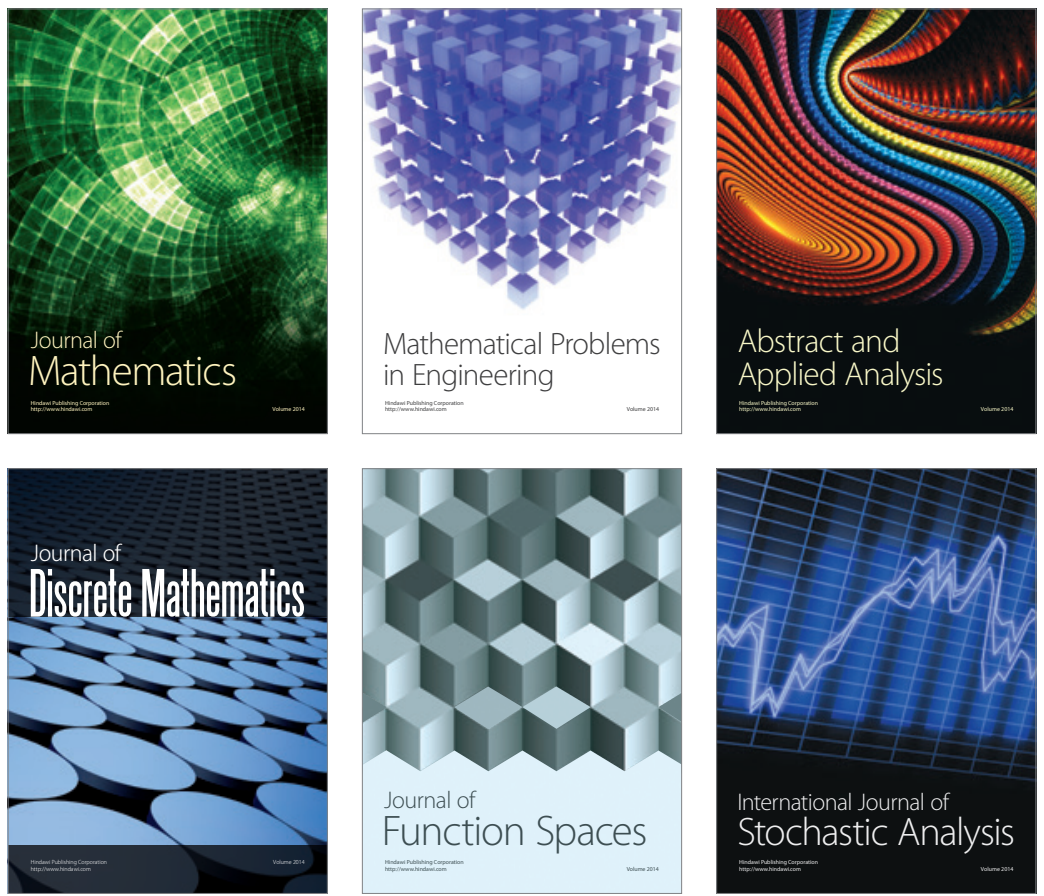

Journal of

Function Spaces

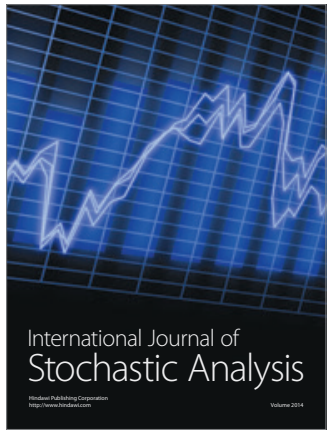

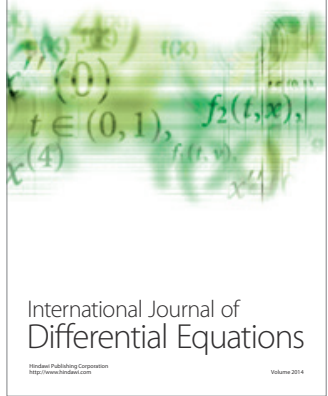
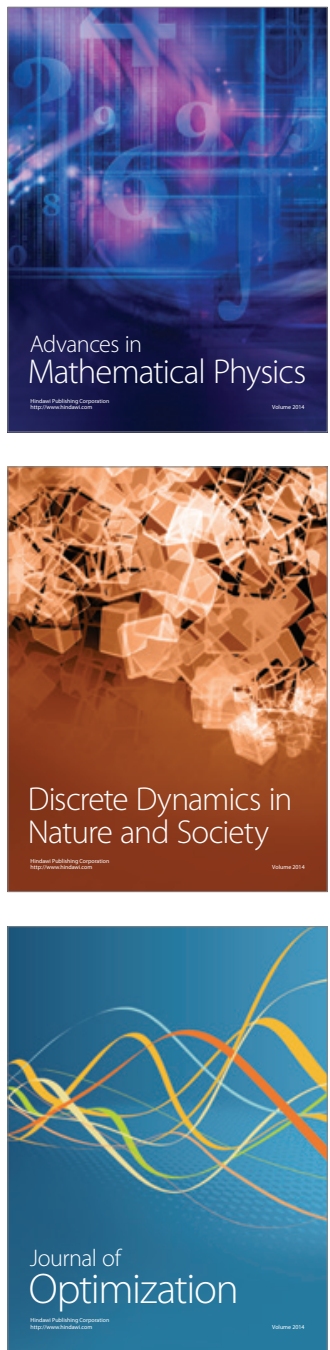